\title{
Exhibition support contents creation at large exhibition museum
}

\author{
Dae-Woong Kim \\ Faculty of Design, Department of Contents Creative Design \\ Kyushu University,Fukuoka,815-8540,Japan \\ Joong-Youp Lee \\ Faculty of Design, Department of Contents Creative Design \\ Kyushu University,Fukuoka,815-8540,Japan \\ Koushi Hoshino \\ Faculty of Fine Art, Department of Photography and Imaging Arts \\ Kyushu Sangyo University,Fukuoka,813-8503,Japan
}

\begin{abstract}
The present research has created and evaluated contents capable of work appreciation visitors changing their viewpoints on their will by introducing mobile exhibition exposition devices (iPad) for large exhibition museum. Various exposition contents of usually invisible parts or those provided in accordance with user positions drew visitors' attentions and improved museum experience satisfaction. Utilization of digitalized exhibition information generated activeness in viewing and new communication between exhibition and a visitor instead of the conventional exhibition exposition.
\end{abstract}

Keywords: behavior appreciation, digital archive, contents design

\section{PREFACE}

Digital archiving of tangible and intangible cultural assets are advancing at museum facilities such as museums and art museums these years. On the other hand, digitalized cultural resources are mainly used for preservation, restoration and data analysis and not actively used to exhibitions. The present research works to create and verify exhibition exposition contents for mobile devices as digital archive utilization. Guide contents utilizing multifunctional mobile devices such as smart phones have probabilities to provide a variety of information by intuitive operation, not conventional one way information. Creation concept of the present research is "to promote behavior appreciation to visitors.” Audio exposition contents heretofore had one to one relationship between object work and exposition. They were not distributing in accordance with visitor behavior or situations, and exhibition style. Large cultural asset exhibitions can often view from all directions as three dimensional exhibitions. We have tried to create guide which can provide detailed information according to angles by professionally researching the object cultural assets. Supposed age ranges are wide and detailed guidance creation is focused

* Corresponding author. E-mail: dwkim@design.kyushu-u.ac.jp Manuscript received Aug 29, 2011 ; accepted Sep.14, 2011 on the points visitors can intuitively enjoy and new interests toward the objects will arouse. Object cultural asset is an important cultural asset stone sekidou pagoda "thousand Buddhas and numerous treasures" possessed by Kyushu National Museum Cultural Exchange Exhibition room. Multiple audio expositions have been created about some characteristics. Viewable places from different angles are identified and navigation starts at the same time so that visitors can appreciate all. Guide contents creation using 3D model data is verified including as well as digital archiving of large tangible cultural asset stone sekidou pagoda "thousand Buddhas and numerous treasures.” This will reveal digital archive utilization stimulation means and new exhibition exposition contents effectiveness.

\section{RESEARCH BACKGROUND}

\subsection{Digital archive}

\subsubsection{Digital archiving at museums}

Digital archiving promotion has been proposed as a method to preserve cultural resources, cultural facilities possessions. Cultural resource digital archiving is a project aimed to pass down precious cultural resource values for a country by digitalizing, saving, accumulating, restoring, and exhibiting using next generation digital technology. Digitalized cultural 
resources can be saved without damages on information for semipermanent and can be sent out to all people regardless of time and space not limited to museum exhibition case thanks to network technology. Many things can be considered as cultural resources. Organizations which fulfill duty to exhibit possessions with cultural values such as libraries, archives, universities, local governments, and corporations are working on cultural resource digital archiving.

Museums including art museums are working very actively among them. Museums are originally institutions for collecting, saving, and exhibiting materials on history, art, folkways, industries, and natural sciences as well as holding programs to earn funds for education, research, and recreation in addition to researching these materials. Museums need to continue collecting and managing materials with high cultural values so digitalization merits are expected to be big.

\subsubsection{Digital archive transitions in Japan}

\section{(1) e-Japan strategy II (the Cabinet Secretariat)}

Japanese government has been working hard on cultural resource digital archiving. An example is "e-Japan strategy II" announced on August, 2003 detailing a scheme, strategy, and policy. It has been promoted not only as a public policy viewpoint insisting that all people should use the public facilities but also as a means to advance understandings in Japanese culture overseas. Below are policies on digital archive aiming at 2005 to realize e-Japan strategy II.

(1)Advance in digital archiving

Take necessary measures to advance digitalization and archiving and to provide information worldwide through the Internet about broadcasting/ publishing, and movie contents, art museums/ museums, library possessions, web information, local culture, official documents about relationships with Asian countries by 2005.

(2) Common index system maintenance

Enrich networks of a nation, local government, private art museums and museums by 2005 in order to make it easier to search digital archive information. Aim to establish portable site about cultural information joined by more than a thousand art museums and museums by 2006.

(3)Technology development for the establishment of image display and transmission technology

Research and develop for the establishment of image display and transmission technology truly reproducing colors, textures, plasticity, and lustrousness of a substance by 2005 in order to smoothly distribute high quality archive.

(4) Technology development for safe and seamless distribution

Promote development and corroborative evidence of technology capable of safe and seamless distribution of archive contents such as the development of unitary high speed searching technology utilizing metadata by 2004 in order to promote contents network use. Likewise, in order to improve understandings of Japanese culture, "IT Strategic Headquarters" of the Cabinet Secretariat has written digital archive promotion and information dispatch worldwide for the first time. IT utilization had been promoted mainly at information about school education and life study among digital archive related policies of priority policy fields.
(2) Cultural heritage online scheme (the Agency for Cultural Affairs)

Based on "e-Japan strategic priority plan - 2003" which summarizes government policies to realize e-Japan strategy II, on April, 2003, the Agency for Cultural Affairs and the Ministry of Internal Affairs and Communications (MIC) have cooperated and announced "Cultural heritage online scheme" aiming to actively disclose information on tangible and intangible cultural heritages through broadband, to protect copyright and to promote utilization. In order to summarize and dispatch information on Japanese cultural heritages, a thousand museums and art museums are hoped to join in fiscal 2006.

On April 2006, the Agency for Cultural Affairs have disclosed test version of Cultural Asset portal site, "Cultural Heritage Online," getting technological cooperation from National Institute of Informatics (Figure 1). It allows searches for 65,000 pieces of cultural asset information registered by a hundred joining cooperation bodies. It is capable of searching for 21,000 pieces of work with image registration and to view them at "GALLERY." Formal version of "Cultural Heritage Online” has been disclosed on March, 2008. "Cultural asset database" has been disclosed to expand cultural asset information from December, 2010.

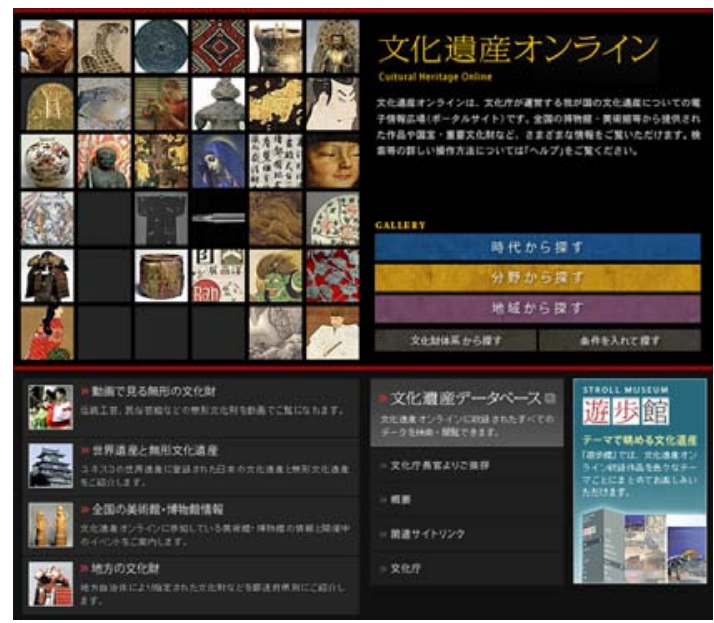

Fig. 1. "Cultural Heritage Online” promoted by the Agency for Cultural Affairs

\section{(3) Local culture digitalization project promotion (MIC)}

MIC has been working on "Local culture digitalization project” from 1999 as cultural resource digital archiving. This project aims to utilize digital image data of tangible and intangible cultural asserts and art works by state-of-the-art digital technology and network technology, to save and success local culture subject to disappear and to enhance information dispatching station environment. It is characterized by prioritizing effective use of digital resources of local culture. Technology development for digitally archived contents utilization is also promoted. Research and development by Japan Science and Technology Agency (JST) is an example of fiscal support. JST Innovation Plaza KYOTO develops large high precision scanner system and image material estimation system. This has enabled two dimensional large cultural assets to be digitally archived and has enabled us to gain information on pigment from data of each element. 


\section{(4) Digital museum scheme}

On September, 2006, the Ministry of Education, Culture, Sports, Science and Technology (MEXT) has started up a study group on digital museum aiming to expand opportunities to experience precious cultural assets by trying to record, save, or restore valuable cultural assets with appreciation restrictions at high-definition graphic by utilizing next generation digital technology. The study group has two policies; one is social innovation by digital image technology development and the other is an international contribution by building systems to dispatch worldwide cultural resources such as art work and academic materials. "Digital museum" is a concept to realize saving, disclosure, and utilization of digitalized cultural resources by the next generation technology, not the conventional digital archiving of museum possessions. We are providing further insights into technological development from next generation digital archving and utilization, distribution, network of archive and into system research and development for "digital museum" corroboration through inspections and opinion exchanges.

\subsection{Current situations and problems \\ 2.2.1 Digital archive popularity}

According to social education research in 2005, digital archive introduction situations are different depending on the running bodies. For example, $13.9 \%$ (17 out of 507) of municipal, $34.4 \%$ (52 out of 151) of prefectural, and $38.5 \%$ (10 out of 26) national facilities have introduced. "Pssession preservation and management” (87.1\%), “curator research” (58.7\%), and "disclosure by the Internet” (58.7 \%) occupy high ratio as digital archive utilization methods (question available for multiple answers). Possession preservation and management, and research belong to the process of "building" digital archives. On the other hand, disclosure on the Internet belongs to "utilization," second use of digital archives. $76.2 \%$ of registered museums and facilities, a thousand and a hundred sixty two in Japan, have their own homepages.

\subsubsection{Problems in digital archiving}

It has only been about 10 years after digital archives are seriously recognized and started. At first, it has been supported as a part of national IT strategy. According to the statistics, at present, the gap is still big between National museums which are likely to have the national support and local museums which are not. So far, it's not proceeding well. It is partly because the government thinks much of the construction of digital archives and little of its utilization. Disclosure on the Internet homepage is considered an effective means to use cultural asset data already saved and maintained. Digital archive utilization needs to be promoted.

\subsection{Preceding research}

\subsection{1 "Bayon Digital Archival Project"}

Ikeuchi laboratory, Institute of Industrial Science, University of Tokyo has digitally archived large tangible cultural asset, Bayon temple in Angkor as "Bayon Digital Archival Project." They called digitalized cultural asset data e-Monument and referred to utilization methods as resources, not only discussing technical issues on digital archives. Preservation and restoration, data analysis, and exhibition are referred to as the main three ways to utilize e-Monument. While preservation and restoration and data analysis are direct use of digitalized data, little research on digital archive application to exhibitions. Indirect utilization contributes a lot to visitors to the museum or the location and is expected to grow. "Bayon Digital Archival Project" is utilized to high definition image contents and user participation contents. The latter enables visitors to appreciate very big scale model and to have new experience such as writing information. This content is characterized by thinking much of reproducibility using rendering algorithm with multiple resolution expression for viewing the model from many viewpoints at real time. Because this requires very advanced computation, it tends to rely on facility equipments. In order to widen digital archive utilization, mobility allowing independent experience from exhibition environment with versatility is required, not only advanced and restrictive contents.

2.3.2 Exhibition exposition support using mobile devices

Museums and art museums have "exhibition exposition" supporting exhibition appreciation. This include graphic panels and captions set around exhibition, kiosk for searching, and theaters where high definition images can be viewed. Besides devices set to exhibition, "mobile audio exposition devices" let visitors enjoy exhibition exposition while walking with ease (Figure 2). Mobile devices have made a huge progress and seem to be more capable to fulfill exhibition exposition role at museums in the future. Although sense of values to museums is in the middle of changing, systematic research has not been implemented heretofore.

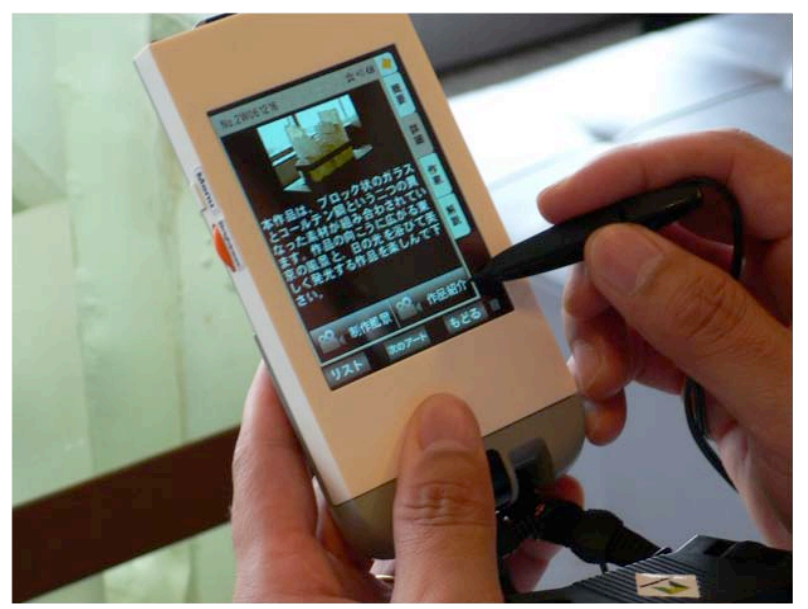

Fig. 2. Mobile devices “ubiquitous art tour”, Tokyo Midtown

\section{RESEARCH OBJECTIVE}

The present research focuses on digital archive utilization problem. Applying digitalized cultural assets to "exhibition," more specifically, "exhibition exposition" at museums may solve the problem. Mobile devices are undergoing rapid technological development. They have interactivity which enables users to enjoy intuitive operations and function as media which enables users to watch three dimensional data and high definition images. Introducing, proposing, and assessing the new technology to museum services will enable us to look for new exhibition exposition contents possibility. The present research aims to propose new solutions for digital archive 
utilization.

\section{CONTENTS DESIGN AND CREATION}

\subsection{Concept}

\subsubsection{Exhibition form}

Kyushu National Museum Cultural Exchange Exhibition room has two exhibition environment forms: "wall exhibition" and "independent exhibition" (Figure 3). Wall exhibition exhibits possessed works divided into detailed themes at each exhibition area each of which represents an era. Independent exhibition is used when exhibiting works with high historical values as cultural assets and large works. While wall exhibitions only have limited viewpoints from one direction, independent exhibition space can be appreciated 360 degrees from all viewpoints.
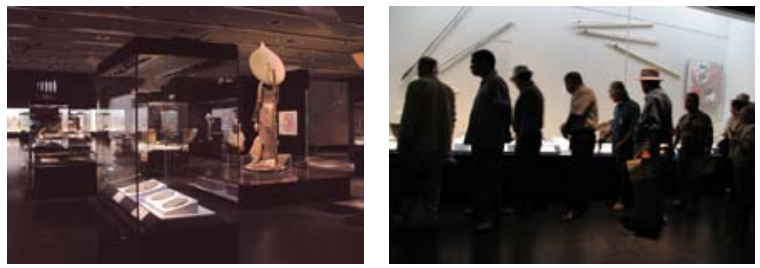

Fig. 3. "Independent exhibition” and "wall exhibition”

Tim Caulton of Sheffield City Industrial Museum in Britain names the conventional traditional exhibition "Hands-off exhibition" and names exhibition form which visitors can directly touch exhibition devices "hands-on exhibition." "Hands-on" has the same meaning with "interactive" and is considered as a method to provide new appreciation experience adopting the newest technology to exhibitions. Interactive exhibition devices aim to help utilization behavior where visitors try to tries to quest to understand the essence of things or phenomena based on their individual selections. The author raises "minds-on" as the goal of new exhibition form. He mentioned that exhibition should aim at designs which move people's mind to quest for the essence of museum materials, not thinking much of physical interactions like interactive exhibition devices. Exhibition exposition is a method to urge visitors' "minds-on." Although every exhibition form exist corresponding exhibition intensions of each piece of possessed work, exhibition exposition only provided the same contents. "Independent exhibition" is available for multiple viewpoint appreciation. If the exhibition exposition also possesses mobility and high functionally devices can be used, interests toward an object will arouse more.

\subsubsection{Behavior appreciation model}

The present research creates contents specialized to exhibition exposition at "independent exhibition." When viewing at "independent exhibition," visitors can change their point of view. However, it depends largely on interests and knowledge of visitors how they appreciate. Exhibition exposition just only provided work abstract. The difference of exhibition forms changes appreciation experience. Promoting "minds-on" of visitors require exhibition exposition design. It is aimed to motivate visitors to "behave" and appreciate in order to utilize the characteristics of independent exhibition, "multiple viewpoint appreciation." The present research names this appreciation model as "behavior appreciation" (Figure 4) and creation concept is "urge visitors to take appreciation behavior.”

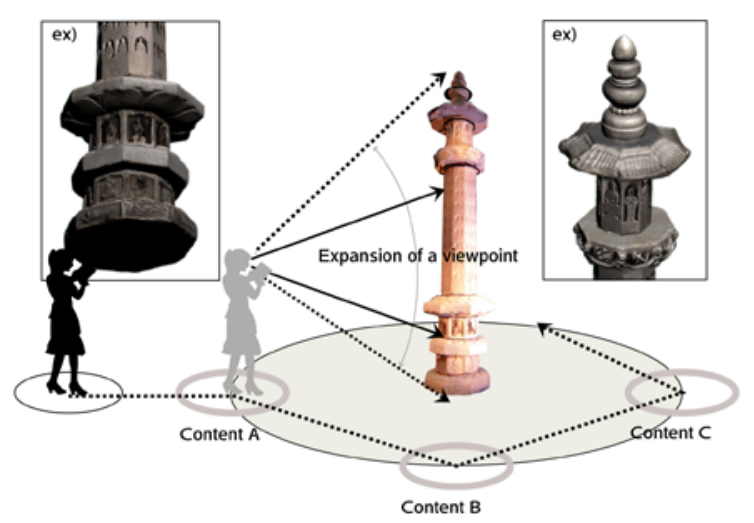

Fig. 4. Image of "appreciation behavior”

\subsubsection{Object cultural assets}

- Important cultural asset "stone sekidou pagoda thousand Buddhas and numerous treasures"

"Stone sekidou pagoda thousand Buddhas and numerous treasures" is a possessions of Kyushu National Museum Cultural Exchange Exhibition room and is an important cultural asset exhibited in "independent exhibition." It is a large cultural asset with hight fifty five meters and total weight thirty five tons donated from Mongolia in 1084 where it was built to Japan.

"Stone sekidou pagoda thousand Buddhas and numerous treasures” was selected for contents creation. Large cultural assets tend to have historical values so museums are considered to be the suitable place to store, preserve, and exhibit. Now large cultural assets digital archives are stored in $3 \mathrm{D}$ models using raiser scanners. When considered from the viewpoint of digital archive utilization, proportionate to the cultural asset size, data quantity also increases a lot when digitalized in high definition. Therefore, many conventional cases provided reproduced contents with large image devices as utilization methods.

The present research proposes mobile exhibition exposition contents application as these large cultural assets digital archive utilization. Large cultural assets are appreciated as "independent exhibition" at many cases so they can attract visitors in many viewpoints.

\subsection{Contents composition}

\subsubsection{Contents composition elements}

Contents composition elements at the present research are roughly divided into "audio exposition" having positioning information and "interface" utilizing 3D data. in order to promote "behavior appreciation" raised at the concept, characteristics of independent exhibition "multiple viewpoint appreciation availability" must be utilized. Audio exposition is created for multiple cases and attaching positioning information to them set them to be played only when visitors move to a certain points. 3D data is used as interface so that visitors are intrigued to find each point. Tablet devices such as 
iPads can allow users to view objects on a relatively big screen and can turn 3D data intuitively by touch operation. Combining this intuitive interface and audio exposition operating together with positioning information will provide visitors new appreciation experience "behavior appreciation." Each composition element is explained hereafter.

\subsubsection{Audio exposition contents}

\section{(1) Abstract}

Creation concept of the present research is "to urge visitors behavior appreciation.” Conventional audio exposition contents have one-to-one relationship between object work and exposition and they were not distributing in accordance with behavior and situations of visitors using exhibition exposition contents, or exhibition form. Large cultural asset exhibitions, object of this time, are very often available for all directions work viewing as three dimensional exhibitions. Researching professionally on the object cultural assets, we tried to make guide which can provide detailed information according to the angles. Visitor age range is big and when creating detailed guide, it can be enjoyed intuitively and focuses on the points which arouse new interests to the objects. Five aural expositions were created for "Stone sekidou pagoda thousand Buddhas and numerous treasures."

Table 1 Audio exposition design corresponding to each sides of stone sekidou

\begin{tabular}{|c|c|}
\hline Number & Audio exposition title \\
\hline 1 & $\begin{array}{r}\text { “Stone sekidou pagoda thousand Buddhas and } \\
\text { numerous treasures.” }\end{array}$ \\
\hline 2 & 「小幢身の逆合掌」 \\
\hline 3 & 「銘文の記憶」“Memory of inscription” \\
\hline 4 & 「天を指す中台」 \\
\hline 5 & 「幢身 96 躯」 \\
\hline
\end{tabular}

Each has detected different angle viewing points and guidance works together with navigation so that behavior to view all will arouse. Below is each exposition.

(2) "Stone sekidou pagoda thousand Buddhas and numerous treasures"

"Stone sekidou pagoda thousand Buddhas and numerous treasures" was made at Liao dynasty in present China about a thousand years ago. Roof, axis, base from the top and each part is clearly separate. It has been clear from research that there is a different China. Serious surface damage let us know that it has always been repaired and preserved heretofore.

The small differences of stone qualities at each part can be observed as well. Each attaching sides have gaps so the axis does not because vertical when connected together. Exhibition inside museums sets exhibitions to be touched at first by visitors and to be seen in vertical. Designing to distribute audio exposition from the adjusted positions, exhibition intention and era background.

\subsection{Creation process}

Creation starts from digitally archiving Stone sekidou using non-touching 3D scanner. 3D data is prepared detailed reproduction of restorations. Exhibition guide system, that is, iPad operation screen interface design, and contents available for each audio exposition listening and screen operation have been created. Exhibition exposition will be provided according to the visitor current position utilizing digital compass which sets terminal directions automatically.

\subsubsection{Digital archives}

Shape data acquisition operation has been conducted for twice using off day of Kyushu National Museum from November, 2008. Main optical measurement device is raiser scanner of three dimensional digitizer Vivid910 of Conica Minorta.. "Stone sekidou pagoda thousand Buddhas and numerous treasures" has $55 \mathrm{~m}$ so large lift is prepared to go up and dow of Vivid910. We have collected all shape as three dimensional data. Work took for about 16 hours with ten people (Figure 5).

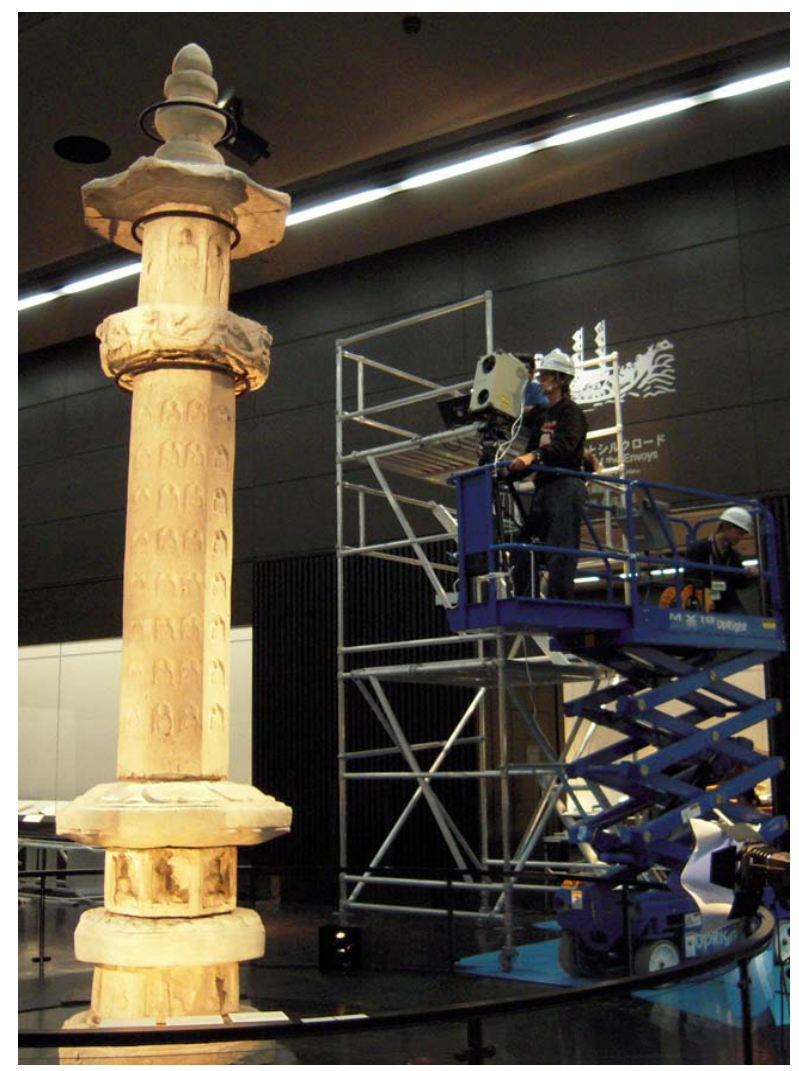

Fig. 5. "Stone sekidou pagoda thousand Buddhas and numerous treasures” and digitalization work

Obtained data when put together had approximately nine million polygon. This is just raw data without digitalization and polygon number has been controlled considering future high vision exhibition image system. iPads or Apple can be considered to have its processing speed slower than expected terminal comparing with desktop PC. Therefore, polygon number of high definition 3D data gained by digitalization need to be restricted and the balance with animation processing speed needs to be adjusted. We processed to maintain smoothness of the shape by using polygon adjustment function of RapidForm and 3ds Max of Autodesk and to apply mesh smooth to low polygon modeling. Decrease in polygon number will reduce surface bumpiness so data visibility also declines. Leaving shape bumpiness as much and mapping processing by data normal line bump map had taken place so that ambience is not lost as digital contents (Figure 6). 


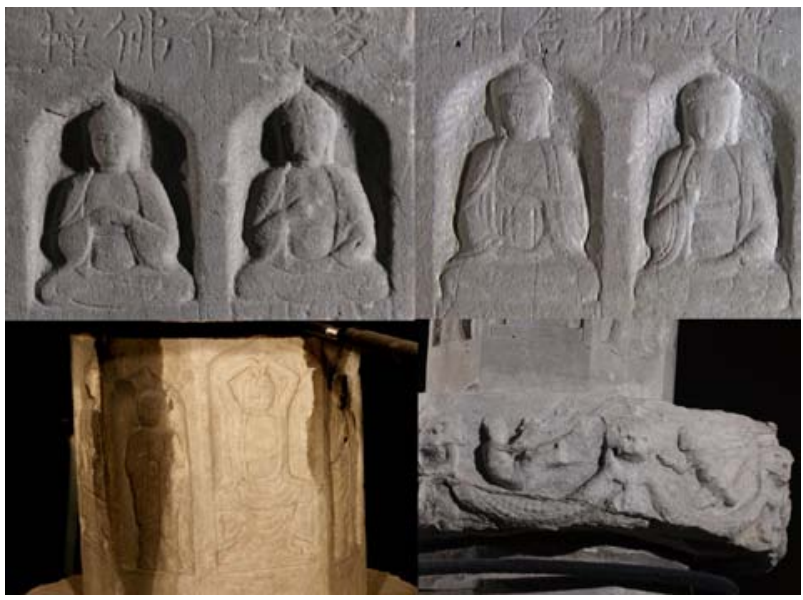

Fig. 6. Still image used for mapping

Normal line bump map is a means to add high definition information on the surface of low polygon objects. It is often used at real time display device such as game engine and is also utilized at rendering scenes or animation. Normal line map is a three color map, not gray scale map used at the normal bump map. Red channel encodes left right axis of normal , green channel encodes vertical information vertical deepness is encoded. Adding normal line map and rendering using 3dsMax will generate modeling data which can maintain interactivity.

\subsubsection{Interface}

\section{(1) Used terminal}

The present research uses iPad of Apple. Conventional exhibition exposition contents devices only provided the minimum of navigation screen. Mobile devices are getting high functions and an example is smart phone. When utilizing digitally archived cultural assets, author thinks that interface is the most effective in exhibition exposition contents. "Audio" is the most appropriate as contents form providing exhibition exposition while viewing but it lacks intuitively enjoyable elements. Putting 3D data in a part of interface which navigates audio exposition contents and if this arouses visitor behavior, new exhibition exposition contents can be provided. An iPad with tablet and mobile will be easy to do interactive operations and digitally archived data can be used a lot.

\section{(2) Operation screen (Figure 7)}

Contents made at the present research are divided into three parts: "360 degree viewing," "exposition,” and "management”. "360 degree viewing” pages include "main operation screen”, "sub operation screen," and "audio exposition navigation" functions which respectively can operate object cultural asset 3D data by touching on the panel, can operate main with button operations, and notifies audio guide distribution corresponding to the visitor position. In order to urge visitors to do "behavior appreciation,” model and exhibition work at the hand of a user (visitor) need to be provided linking form and angles. We have designed the device to have three positioning information, 1)visitor, 2) object cultural asset $3 \mathrm{D}$ model, and 3) object cultural asset, and to make them function together. "Exposition" page shows five playable audio exposition text base after clicking “360 degree viewing.”

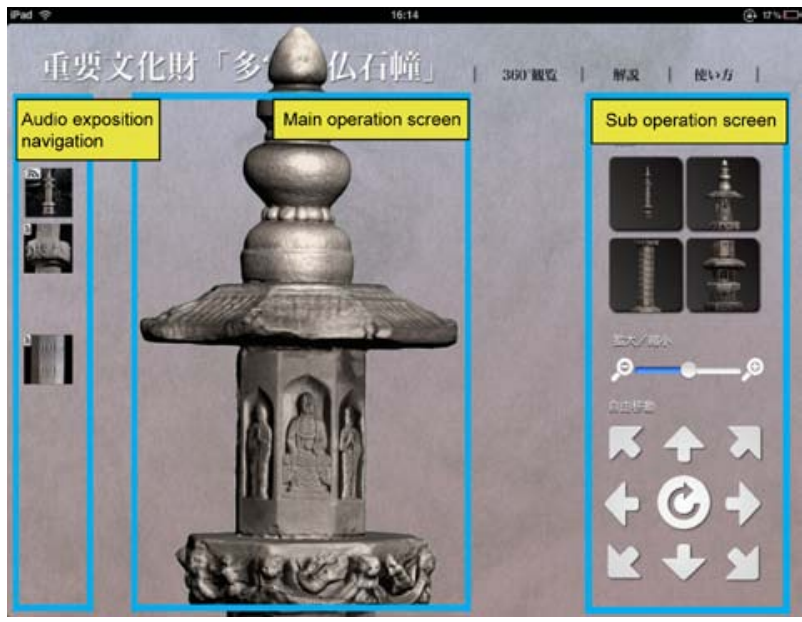

Fig. 7. Exhibition exposition device (iPad) interface

(1) Main operation screen

Digitally archived 3D data is intuitively operated by touching. Installed functions are free viewpoint change, magnify/minimize, and position relation (contents and exhibition space). When viewing large cultural asset, some places cannot be seen directly with eyes. On the other hand, indirect viewing from free viewpoints by $3 \mathrm{D}$ data will enable viewing in accordance with visitor interests from all direction. Angle from the above or a new discovery will maintain high motivation to play audio exposition contents.

(2) Sub operation screen

Sub operation screen can change touch input to button input taking place at main operation screen. Operation panels are divided into three: viewpoint panel, magnify/minimize panel, and free mobile panel. This is aimed for users who are not used to touch interface of table terminals. It has choices on input operation for a usability design.

-Viewpoint panel

Viewpoint panel has four buttons (Figure 8). From upper left to the whole, upper magnify, lower magnify, center magnify and upper and lower magnify buttons are for the needs to watching places where users cannot directly see.
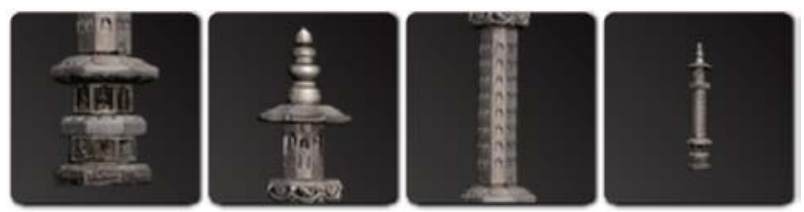

Fig. 8. Each button for viewpoint panel

\section{-Magnify and minimize panel}

This panel sets minimum to $100 \%$ scale and can magnify enough to watch the details of each angle. The advantage of digital archives is that data of detailed reproduced bumping can also be obtained. Direct viewing allows users to view in physically restricted angle. Detailed guide by navigating audio exposition contents will arouse new interests of users.

-Free moving panel

Free moving panel supports circulation operation and upward and downward angle changes. Angles have restrictions because 3D modeling underwent rendering as prototype is used 
as animation base. Therefore, it has been designed to smoothly change to the next angles by button input operation.

(3) Audio exposition navigation

Aural exposition navigation is a panel visualized "position link" function at interface. The present research aims to promote behavior appreciation of visitors by exhibition exposition contents. "Position link" equipped as interface function can fulfill navigation role distributing five audio exposition contents linked with angles and positions a user is watching at the object both of which will be identified by digital compass. One piece of work corresponds to one exposition and contents used to abstract unreliable to exhibition space most times. However, exhibition work differs drastically depending on the object cultural assets. Three dimensional and large cultural assets can be viewed from all directions so grasping the behavior history and providing expositions for each angle will arouse new interests.

"Digital compass" is a characteristic sensor of iPad. Operating 3D data, the base of interface, and user angle together will enable users to receive navigate from many viewpoints. Audio exposition corresponding to angles will induce users to watch in all angles and to figure out something new about the work. Audio exposition corresponding to angles is also synchronized to positioning information and five panels of "audio exposition navigation" are designed to flash according to user positions (Figure 9). Sending out the signal to visitors can urge behavior appreciation.

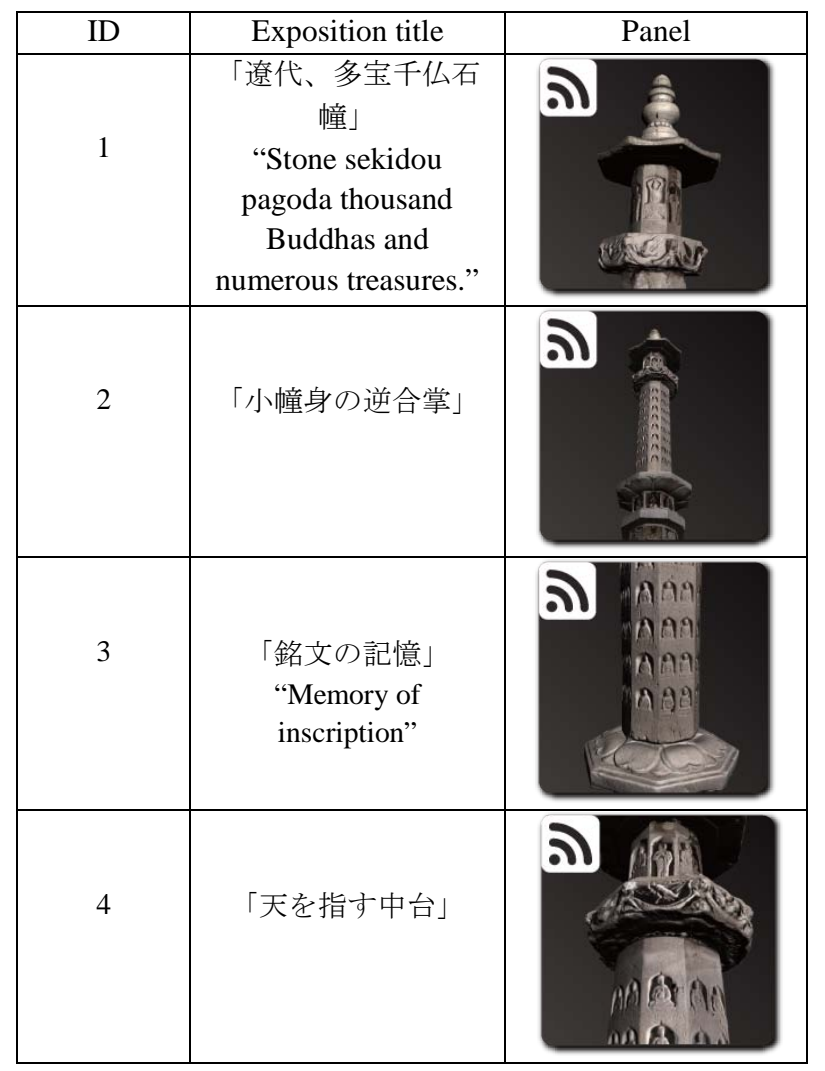

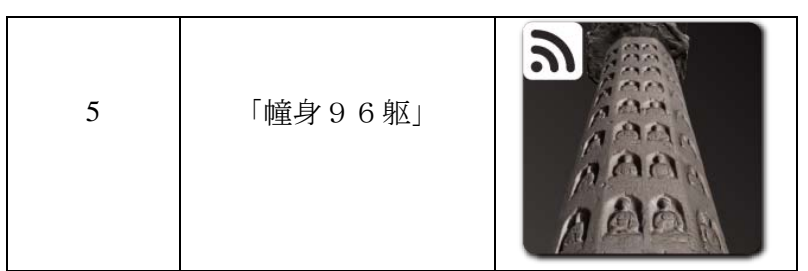

Fig. 9. A panel displayed at "audio exposition navigation”

\subsubsection{Image data processing}

Contents for mobile devices like iPad priorities usability. It is difficult to deal with digitally archived three dimensional modeling data because data processing speed is slower than desktop computers. The present research uses rendering data of high resolution modeling data of 3D Studio Max as prototype. We used a system which falsely enables each function operation by programming processing using still image sequence in accordance with processed contents. "Free viewpoint movement" and "magnify/minimize" function is equipped as interface. In order to falsely reproduce this, seven "angles" on the vertical direction of objects and sequences which rotate one cycle at thirty six frames although an arbitrary rotation axis cannot be taken. Sequences with different resolutions are also set in order to make it possible to have four levels of "magnify/minimize." This enabled to maintain appropriate operation and stable resolution.

Table 2. Touch operation corresponding to function

\begin{tabular}{|c|c|}
\hline Function & Touch operation \\
\hline Free viewpoint movement & Swipe \\
\hline Magnify/Minimize & Pinch \\
\hline Position link & Digital compass \\
\hline
\end{tabular}

\subsubsection{Digital compass processing}

Contents created at the present research obtain positioning information of visitors using digital compass. Suppose that object cultural assets are in the center of round "independent exhibition” space. Device directions held by visitors are gained as figures at digital compass. As the same with touch event processing, it is designed to be converted into image file ID allocated in the range and visitors positions are synchronized with 3D model on the display and with object cultural asset directions in the exhibition space. When a value is judged to be in a certain range, "audio exposition" will be distributed. Visitors are urged find out a certain position following audio exposition navigation and can intuitively do behavior appreciation (Figure 10). 


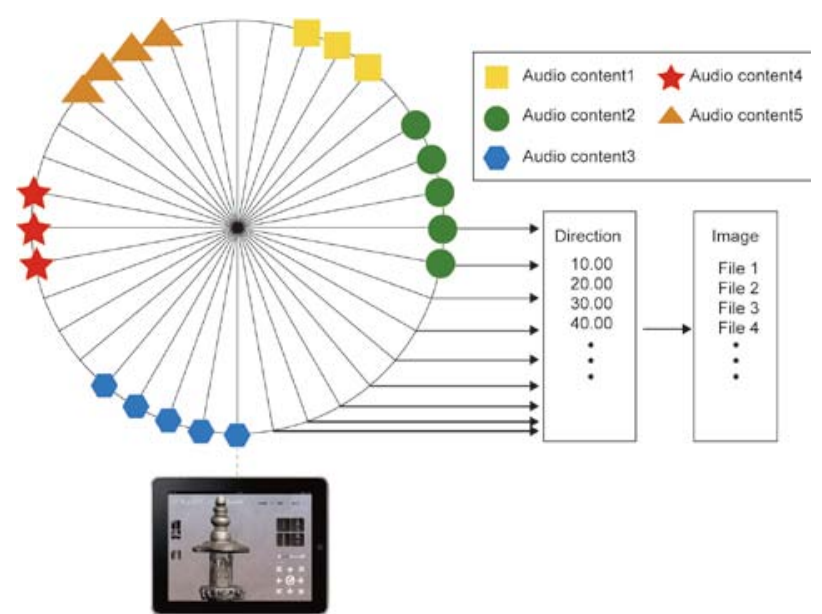

Fig. 10. Digital compass processing

\section{EVALUATION}

\subsection{Evaluation objective}

The present research aims to urge visitor behavior appreciation and to contribute to museum experience value improvement by exhibition exposition contents to be proposed. We have searched for contents satisfaction in order to measure the effect. Interface using $3 \mathrm{D}$ data is proposed in order to promote "behavior appreciation." This usability is also assessed. Evaluation objectives are the following.

\section{(1) Interface usability}

The present research adopts interface which can unrestrictedly operate 3D data of digitally archived cultural assets with touch panels. Unrestricted operation include "magnify/minimize," “viewpoint change," "rotation,” and "positioning link by digital compass." Questionnaire has been conducted to evaluate each operation and interface usability is assessed from the result.

\section{(2) Satisfaction in overall contents}

This exhibition exposition contents are designed to distribute five audio expositions cooperated with positions in addition to the aforementioned interface. When considering satisfaction in audio exposition, visitor behavior can be influential so conduct verification including usability and see satisfaction in contents overall.

Paying attention to the above, discuss visitor "behavior appreciation" and verify what kind of changes were brought in into appreciation experience in museums and if the present research was appropriate.

\subsection{Evaluation methods}

The present research is conducted as a part of "Personal museum concierge," joint research with Kyushu National Museum. Last year, corroborative experiment has been conducted against general visitors to Cultural Exchange Exhibition room for about a week from December 14 (Figure 11). Evaluation is based on questionnaire conducted to 31 people who visited "Stone sekidou pagoda thousand Buddhas and numerous treasures" and used exhibition exposition contents.

o Date: December 14, 2010—December 19, 2010

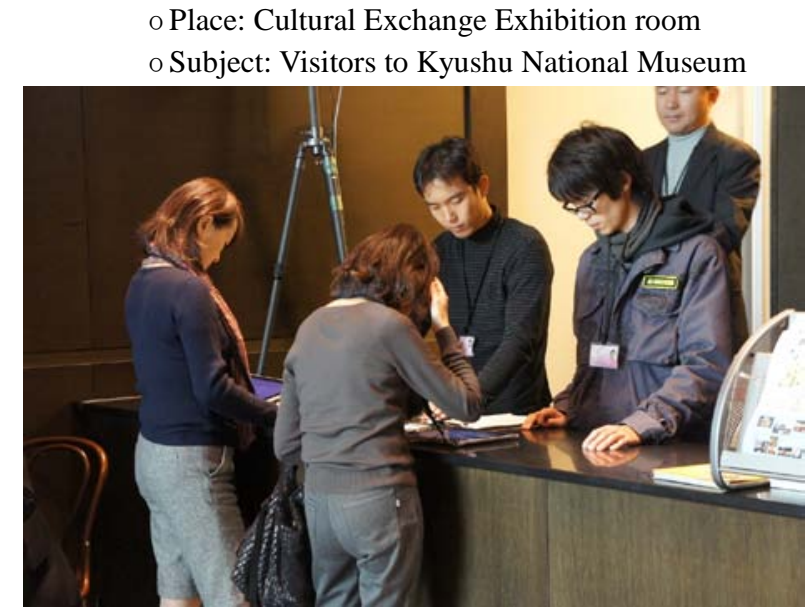

Fig. 11. Corroborative experiment toward general visitors

\subsection{Result and Discussion}

Questionnaire has been carried out as corroborative experiment with Kyushu National Museum renting exhibition exposition contents on "Stone sekidou pagoda thousand Buddhas and numerous treasures" to general visitors. After using the contents, thirty one subjects evaluated. Question 1 asked mobile touch panel use experience. Those who answered they have experienced using touch panels amounted to $65 \%$ (20 people). These kinds of people who have device literacy or interested in the devices seem to have some kind of interests in exhibition exposition using new mobile devices. Question 2 asked for the usability of touch panels. Eleven people answered "easy to use" and thirty people answered "easy to use to some degree”, which means $78 \%$ of all have responded preferable answers. Devices with touch panels just came out so people may have had resistance or difficulty in use but it seems almost no problem exist. Question 3 asked if "magnify/minimize" operation is useful to appreciation. "magnify/minimize" operation function on touch panel was "useful" for seventeen people and "so-so useful" for eleven people. Some exhibition works are not available for touching or getting close. 3D data utilization comfortably conducts this operation, which can be expected to raise appreciation experience value. Question 4 asked if "viewpoint change" operation is useful for appreciation (Figure 12).

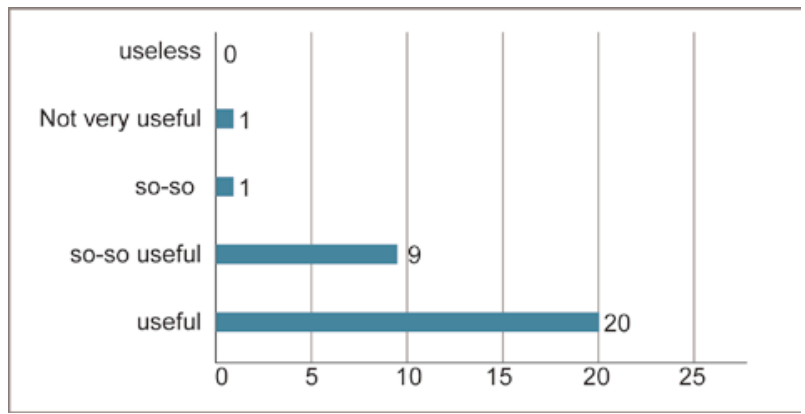

Fig. 12. Was "viewpoint change” operation useful for appreciation?

Twenty people answered "useful" and nine people answered "so-so useful," $94 \%$ of all. When viewing large work, it is difficult to see the upper part or detailed decorations. 
Combining "viewpoint change" and "magnify/minimize" operations will easily provide detailed information on upper and lower parts, which will raise satisfaction in appreciation. Question 5 asked if "rotation" operation is useful for appreciation. While nineteen people answered "useful," which is $61 \%$ of all, other subjects have discrepancies in their opinions. "Rotation" operation can be manually changed by visitors using a touch panel. Position link using "digital compass" can automatically change as well. No restrictions to rotation and if there is no input it is set to rotate gradually linking with current location but this gap adjustment seemed to puzzle some visitors. This is a problem. Question 6 asks if "digital compass" function is useful to appreciation which links visitor position and work directions (Figure 13).

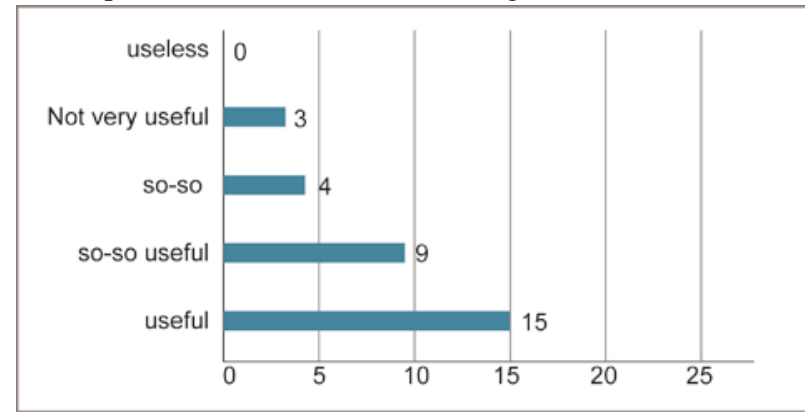

Fig. 13 Was digital compass function useful for appreciation?

Fifteen people answered "useful" and nine people answered "so-so," which is $77 \%$ out of all. No preceding cases where digital compass has been utilized to contents in exhibition exposition field. It was a new experience for visitors so some people looked perplexed and some were surprised but most people had preferable opinions about operation. It is not a difficult operation but an intuitive function linked with their own behavior so most people has got used to it as time passes. On the other hand, those who could not get used to it had lower "rotation" operation satisfaction as well. More improvements are required. Question 7 asked how the focus on exhibition was while listening to audio exposition. Eleven people answered "good focus" and nine people answered "so-so." $64 \%$ people had preferable opinions and $13 \%$ were dissatisfied, which can infer that the contents of this time cannot really be an obstacle at real work appreciation. Question 8 asked if interests into works have increased after listening to audio exposition. Eighteen people have answered their interests arouse through audio exposition and eight people have answered "so-so." $78 \%$ of all have answered that guide triggered arousal of interests in works. Contriving and implementing many ideas so that visitors can have new discoveries will increase their interests in museums. One of those who answered "I got interested" said "there was no order for audio exposition so it may be better to have a program which enables people to listen all in an order.” Five audio expositions were provided this time but they had no relationship with each other or were not based on the idea to be chronologically provided. Not only entrusting the playing order to visitors, we would like to consider creating a program for "behavior appreciation" with story and add one to contents. Seventeen people answered they "would like to use" $3 \mathrm{D}$ contents again in the future and twelve people answered "if there are, I would like to use," both of which totalizes to approximately $90 \%$ of all. Contents details need to be improved a lot but it seems subjects got satisfied to a certain extent through new appreciation experience (Figure 14).

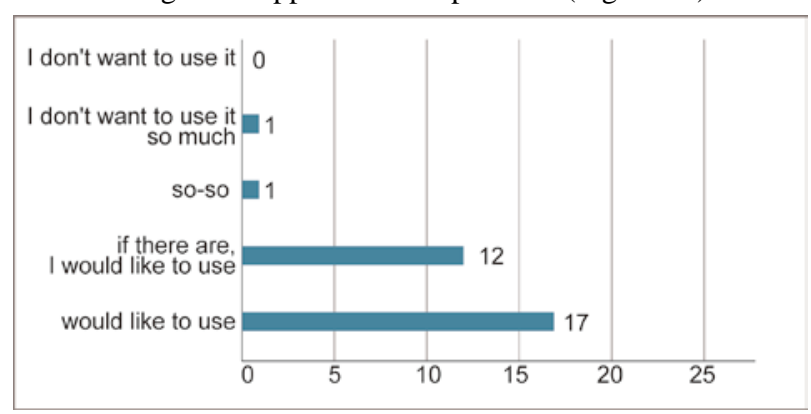

Fig. 14. Would you like to use 3D contents again while viewing?

Based on the above questionnaire, we would like to consider interface usability and satisfaction in overall contents.

-Interface usability

It was first time for about half of the subjects to use tablet devices at the experiment. About $80 \%$ of subjects have answered that basic operations such as "magnify/minimize," "viewpoint change," and "rotation" are easy to use, intuitive interface design will make new devices usable in an appropriate operation. On the other hand, "rotation" received lower evaluation than "magnify/minimize" and "viewpoint change" most likely due to digital compass utilization. Prioritizing the link of visitors, model on the display, and object cultural assets will make the device synchronize with visitor location after touch operation processes. We discovered that visitors think events (image processing change) occurred by force and this has possibility to make them anxious in operation.

-Satisfaction in overall contents

Contents created at the present research provide audio guide with positioning information. Audio guide received preferable evaluation in understanding of the work, interests, and increased motivation. Subjects who are highly satisfied with interface gave high evaluation to contents as well. When conducting "positioning link" of a visitor and object cultural assets through a mobile device, there are not clear borderline between interface and contents so it is desired that both will function well. The verification of this time showed that it is able to make the audio exposition quality higher according to objects but we also need to consider this does not become obstacles for real work appreciation. We are required to fulfill the role of "exhibition exposition" to support exhibition appreciation utilizing the flexibility of setting audio exposition points.

\section{SUMMARY}

\subsection{Conclusion}

The present research created and verified exhibition exposition contents for new mobile devices. We proposed interactive contents which can obtain visitor location using "digital compass" and provide audio exposition at multiple 
viewpoints, not like the conventional one-way guide. We have verified at the view point of 1) interface usability and 2) satisfaction in overall contents from research results. Approximately $80 \%$ of subjects have evaluated that interface is "easy to use" from basic operations to newly tried positioning information synchronization regardless of visitor device use experience. Satisfaction in overall contents was also high at any points, understandings to the work through audio guidance, interests, and increased motivation. Therefore, it can be concluded that we were somehow successful to reach out research objective, to promote "behavior appreciation" and to arouse visitors' interests in work by new appreciation experiment.

\subsection{Problems from now on}

Affordance toward "positioning link" is a problem we need to tackle. Contents created this time automatically processes user position information, which let some subjects to become anxious. We need to provide processing feedback in a clear way for especially those who are new to table devices. It is indispensable to utilize objective exhibition evaluation system as user feedback methods. The concept of the present research, "behavior appreciation," requires objective analyses by numerating what kind of contents visitors played and how far they moved around in the exhibition space, besides subjective contents satisfaction. Highly functionalized devices such as iPad can record these kinds of figures as log. Exhibition exposition contents should be utilized as a tool to newly discover needs against exhibition by recording, saving, and analyzing visitor appreciation history in detail.

\subsection{Future prospect}

Digital archiving of cultural assets are steadily taking place worldwide. However, application to "exhibition" only has a limited number of examples although exhibition contributes the most to people among preservation and restoration, data analysis, and exhibition. This raises an issue that enormous amounts of archived data are not fully utilized.

New utilization possibility of digital archives does not depend on the "quantity" of digitalized cultural asset data. The original exhibition values lies on providing meeting scarce exhibit as intellectual experience. Guide contents using mobile devices are in the role to support this exhibition role, which are considered to be new digital archive utilization. In order to provide contents as mobile media, it is indispensable to link interface with user situations. It is important to verify contents style adaptable to developing mobiles and to seek for digital archive utilization.

\section{REFERENCE}

[1] Katsushi Ikeuchi,Daisuke Miyazaki ,Digitally Archiving Cultural Objects, Springer-Verlag New York Inc (C), 2007.

[2] Tim Caulton, Hands-on exhibitions,Tokai University press, 1988.

[3] Inoue tadashi, Stone sekidou pagoda thousand Buddhas and numerous treasures, Kyoto National Museum.2004

[4] Ministry of education, culture, sports, science and technology-japan ,MEXT White paper, 2007
[5] Katsushi Ikeuchi, Takeshi Oishi, The Great Buddha Project:Digitally Archiving, Restoring, and Analyzing Cultureal Heritage Objects, International Journal of Computer Vision, vol.75, no.1,2007, pp.189-208.

[6] Joongyoup Lee,Daewoong Kim, Developing and Evaluation of Visitor's Behavior Evaluating System for Exhibition Facilities, JSSD, vol55,no.4,Oct. 2008. pp.77-86.

[7] Bayon Digital Archival Project, http://www.cvl.iis.u-to kyo.ac.jp/research/bayon/jp/project.

[8] Institute of Industrial Science,The University of Tokyo Ikeuchi Lab,http://www.cvl.iis.u-tokyo.ac.jp/projects.html

[9] Cultural Heritage Online, http://bunka.nii.ac.jp/Index.do

[10] Cultural Heritage Database,http://bunka.nii.ac.jp /db/ Index.do.

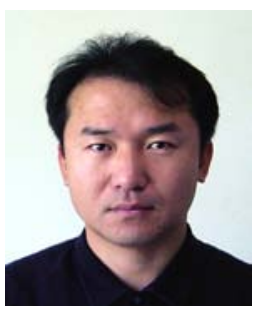

\section{Dae-Woong Kim}

Faculty of Design, Kyushu University Completed master's course at Graduate School of Design, Kyushu Institute of Design in 1998. An instructor at Department of Art and Information Design, Kyushu Institute of Design, from 2002.An associate professor at Department of Art and Information Design, Kyushu Institute of Design, from 2005 till now. Engage in research on digital contents and digital archive. A member of JSSD, the Japan Society for Exhibition Studies, Design Research Association, ADADA.

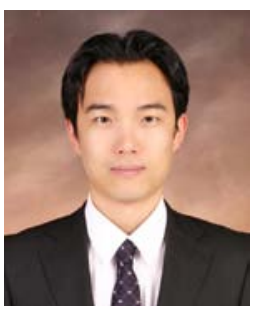

Joong-Youp Lee

Faculty of Design, Kyushu University

Completed master's course at Graduate School of Design, Kyushu University in 2006.

Completed doctoral course in 2009.

Postdoctoral academic researcher at Department of Contents Creative Design, Kyushu University from 2009 till now. Engages in research on contents design, interface design and etc. A member of JSSD, Design Research Association, ADADA.

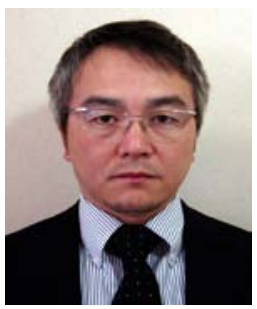

\section{Koushi Hoshino}

Completed doctoral course at Graduate School of Design, Kyushu University in 2010.

Currently associate professor at Faculty of Fine Art, Department of Photography and Imaging Arts, Kyushu Sangyo University. Engage in research on contents effective use at 3DCG, digital contents, and digital museum. A member of Japanese Society for the Science of Design (JSSD), Japan Museum Management Academy (JMMA), the Society of Photographic Science and Technology of Japan (SPSTJ), Asia Digital Art \& Design Association (ADADA). 